\title{
Effects of breathing supplemental oxygen before progressive exercise in patients with chronic obstructive lung disease
}

\author{
JAMES L MCKEON, KEITH MURREE-ALLEN, NICHOLAS A SAUNDERS \\ From the Department of Thoracic Medicine, Royal Newcastle Hospital, and the Discipline of Medicine, \\ University of Newcastle, New South Wales, Australia
}

\begin{abstract}
A study was carried out to determine whether supplemental oxygen before exercise would improve maximum exercise performance and relieve exertional dyspnoea in 20 patients with chronic obstructive lung disease (mean $\mathrm{FEV}_{1} 0.79$ l; forced vital capacity 2.30 l). Patients performed two progressive treadmill exercise tests to a symptom limited maximum, with at least 30 minutes rest between tests. They received compressed air or supplemental oxygen from nasal prongs for 10 minutes before exercise in a double blind randomised trial with a crossover design. Heart rate and breathlessness score on a visual analogue scale were compared between tests at $75 \%$ of the maximum distance walked in the compressed air test. The mean arterial oxygen saturation $\left(\mathrm{SaO}_{2}\right)$ after oxygen $(93 \%)$ was significantly higher than after compressed air $(91 \%)$. There was no significant change, however, in maximum distance walked or maximum heart rate, or in the breathlessness score or heart rate at $75 \%$ of maximum distance walked. The study had a power of $93 \%$ for detecting an increase of 50 metres in maximum distance walked. There was an order effect, with better performance on the second test; but the magnitude of the difference was small. It is concluded that administration of supplemental oxygen sufficient to raise $\mathrm{SaO}_{2}$ above $90 \%$ for 10 minutes before exercise is unlikely to improve maximum exercise performance or breathlessness on exertion in patients with chronic obstructive lung disease.
\end{abstract}

Supplemental oxygen improves exercise performance and relieves dyspnoea when used during exercise by patients with chronic obstructive lung disease..$^{1-4}$ Portable oxygen, however, is inconvenient and expensive and the extra weight carried by the patient sometimes outweighs the gains from oxygen. ${ }^{3}$ There is conflicting evidence on whether supplemental oxygen before exercise improves exercise performance and exercise related dyspnoea. In 1981 Woodcock et al showed benefit from pre-exercise oxygen in 10 patients with chronic obstructive lung disease, and this has been cited in a recent review of treatment for chronic bronchitis and emphysema. ${ }^{5}$ Rhind et al, ${ }^{6}$ however, recently failed to show improvement in distance walked or in breathlessness in 12 patients with chronic bronchitis when "predosed" with supplemental

Address for reprint requests: Professor N A Saunders, Room 340. David Maddison Clinical Sciences Building, Royal Newcastle Hospital, Newcastle, New South Wales 2300, Australia.

Accepted 13 July 1987 oxygen before exercise. We have therefore conducted a double blind trial of oxygen and compressed air given before a progressive maximal exercise test in 20 patients with chronic obstructive lung disease, to determine whether "predose" supplemental oxygen improves maximal exercise performance and relieves breathlessness during exercise by comparison with placebo.

\section{Methods}

We studied 20 consecutive patients with chronic obstructive lung disease referred for assessment of their need for home oxygen therapy. All patients gave informed consent. Patients had chronic airflow obstruction $\left(\mathrm{FEV}_{1} /\right.$ forced vital capacity $(\mathrm{FVC})<60 \%$, total lung capacity (TLC) $>80 \%$ predicted), with no significant change after bronchodilator. Patients were not studied during exercise if there was a history of angina, recent myocardial infarction, exercise induced syncope, or left ventricular failure. All patients under- 
went routine pulmonary function testing, including measurement of lung volumes by body plethysmography and carbon monoxide gas transfer (TLCO) by a single breath method. Spirometry was measured before and 15 minutes after $5 \mathrm{mg}$ nebulised salbutamol. Arterial blood was drawn from the radial artery, while the patient was seated after resting for 15 minutes, and analysed for $\mathrm{pH}$, oxygen tension $\left(\mathrm{Po}_{2}\right)$ and carbon dioxide tension $\left(\mathrm{PCO}_{2}\right)$ with standard electrodes (Radiometer ABL-2). In addition, arterial oxygen saturation $\left(\mathrm{SaO}_{2}\right)$ was measured with an ear oximeter (Biox 3700, Ohmeda, Colorado). If the resting $\mathrm{SaO}_{2}$ was under $90 \%$, the oxygen flow rate required to achieve an $\mathrm{SaO}_{2}$ of at least $90 \%$ was recorded. The haemoglobin concentration, chest radiograph, and electrocardiograph were assessed before study.

Patients were randomised to receive compressed air or supplemental oxygen by nasal prongs at $2.51 \mathrm{~min}{ }^{1}$ for 10 minutes before the first exercise test. This flow rate of oxygen was enough to raise resting $\mathrm{SaO}_{2}$ to at least $90 \%$ in all patients when seated. Neither the patient nor the operator knew whether compressed air or supplemental oxygen had been given. Patients were told that both cylinders contained oxygen, but in different concentrations. All patients had done at least one practice walk on the treadmill before the study. Each patient performed two progressive exercise tests on the treadmill (Repco Equipment Company, Sydney) until limited by symptoms, with at least 30 minutes' rest between each test. The gradient was kept flat throughout the test. The starting speed was $1 \cdot 5$ $\mathrm{km} / \mathrm{h}$ with increments of $0.5 \mathrm{~km} / \mathrm{h}$ every minute. All patients exercised to their maximum and all were limited by dyspnoea. During exercise the heart rate and oxygen saturation were monitored by ear oximetry (Biox IIA, Ohmeda, Colorado). Breathlessness was assessed by a $300 \mathrm{~mm}$ visual analogue scale,? scores being recorded before and each minute during exercise. The maximum distance walked was recorded for each test. Heart rate and visual analogue scale scores were compared at a distance equal to $75 \%$ of the maximum distance walked with compressed air (VAS75, HR75).

Comparisons between tests were made by paired $t$ tests. The power of the study was calculated by a standard formula. ${ }^{8}$

\section{Results}

Of the 20 patients, 13 were men and seven women. Fourteen had physiological evidence of emphysema, two had bronchiectasis, and four had chronic bronchitis. Table 1 shows anthropometric data and pulmonary function for the group.

Table 2 shows results of the exercise tests. The mean
Table 1 Anthropometric data and pulmonary function in 20 patients with chronic obstructive lung disease

\begin{tabular}{|c|c|c|c|}
\hline & Mean & $S D$ & Range \\
\hline $\begin{array}{l}\text { Age (years) } \\
\text { Height (cm) } \\
\text { Weight (kg) } \\
\text { FEV (l) } \\
\text { FEV, (\% pred) } \\
\text { FVC (1) } \\
\text { FVC (\% pred) } \\
\text { RV (\% pred) } \\
\text { TLC (\% pred) } \\
\text { TLCO (\% pred) } \\
\text { pH } \\
\mathrm{PaO}_{2}(\mathrm{~mm} \mathrm{Hg}) \\
\mathrm{PaCO}_{2}(\mathrm{~mm} \mathrm{Hg}) \\
\mathrm{HCO}_{3}(\mathrm{mmol} / \mathrm{l}) \\
\mathrm{Oximeter} \mathrm{SaO}_{2}(\%)\end{array}$ & $\begin{array}{l}63 \cdot 2 \\
165 \\
62 \\
0 \cdot 79 \\
31 \\
2 \cdot 30 \\
68 \\
206 \\
122 \\
55 \\
7 \cdot 43 \\
58 \\
44 \\
28 \cdot 8 \\
90\end{array}$ & $\begin{array}{l}10 \\
9 \\
14 \\
0 \cdot 29 \\
13 \\
0 \cdot 70 \\
17 \\
60 \\
24 \\
32 \\
0 \cdot 04 \\
9 \\
9 \\
5 \cdot 6 \\
3\end{array}$ & $\begin{array}{l}42-74 \\
150-182 \\
38-94 \\
0 \cdot 45-1 \cdot 50 \\
17-62 \\
1 \cdot 00-3 \cdot 85 \\
35-121 \\
100-334 \\
80-183 \\
6-120 \\
7 \cdot 35-7 \cdot 56 \\
43-82 \\
31-62 \\
21 \cdot 8-39 \cdot 6 \\
84-96\end{array}$ \\
\hline
\end{tabular}

Conversion: Traditional to SI units-Blood gas tensions: $1 \mathrm{~mm} \mathrm{Hg}$ $=0.133 \mathrm{kPa}$.

FVC - forced vital capacity; RV - residual volume; TLC - total lung capacity; TLCO-carbon monoxide transfer factor; $\mathrm{PaO}_{2}-$-arterial oxygen tension; $\mathrm{PaCO}_{2}$-arterial carbon dioxide tension; $\mathrm{HCO}_{3}$ plasma bicarbonate; $\mathrm{SaO}_{2}$-arterial oxygen saturation.

(SEM) $\mathrm{SaO}_{2}$ at rest before exercise was higher after oxygen $(93 \%(1 \%))$ than after compressed air $(91 \%$ $(1 \%))(p<0.01,95 \%$ confidence limits of the difference 1-3\%). The end exercise $\mathrm{SaO}_{2}$, however, was not significantly different, nor was there any significant difference in maximum distance walked, VAS75, or HR75 between compressed air and oxygen. There were no significant differences in mean visual analogue scale scores at any time during exercise between the compressed air and oxygen tests (fig 1). There was also no significant difference in mean heart rate between the compressed air and oxygen tests at any time.

When the group was stratified according to resting $\mathrm{SaO}_{2}$ and $\mathrm{PaCO}_{2}, 11$ patients had a resting $\mathrm{SaO}_{2}$ above $90 \%$ and eight patients had a resting $\mathrm{PaCO}_{2}$ of $43 \mathrm{~mm}$ $\mathrm{Hg}(5.7 \mathrm{kPa})$ or more. Neither resting $\mathrm{SaO}_{2}$ nor $\mathrm{PaCO}_{2}$ altered the finding of no significant difference between the compressed air and oxygen tests in maximum distance walked, VAS75, and HR75.

Table 2 Results (means with standard errors in parentheses) of exercise tests performed after compressed air ( $\mathrm{CA}$ ) and supplemental oxygen $\left(\mathrm{O}_{2}\right)$

\begin{tabular}{lll}
\hline & $C A$ test & $O_{2}$ test \\
\hline Resting $\mathrm{SaO}_{2}(\%)$ & $91(1)$ & $93(1)^{*}$ \\
End exercise SaO, $(\%)$ & $83(2)$ & $84(2)$ \\
DISTmax (m) & $272(40)$ & $274(35)$ \\
VAS75 (mm) & $182(9)$ & $182(16)$ \\
HR75 (beats/min) & $123(4)$ & $122(4)$ \\
HRmax (beats/min) & $127(5)$ & $129(4)$ \\
Exercise duration (min) & $5 \cdot 5(0.6)$ & $5.7(0.5)$ \\
\hline
\end{tabular}

*Significantly different from CA by paired $t$ test; $p<0.01 ; 95 \%$ confidence limits of difference in $\mathrm{SaO}_{2}=1-3 \%$.

$\mathrm{SaO}_{2}$-arterial oxygen saturation; DISTmax - maximum distance walked; HR 75 - heart rate at $75 \%$ of the maximum distance walked. 


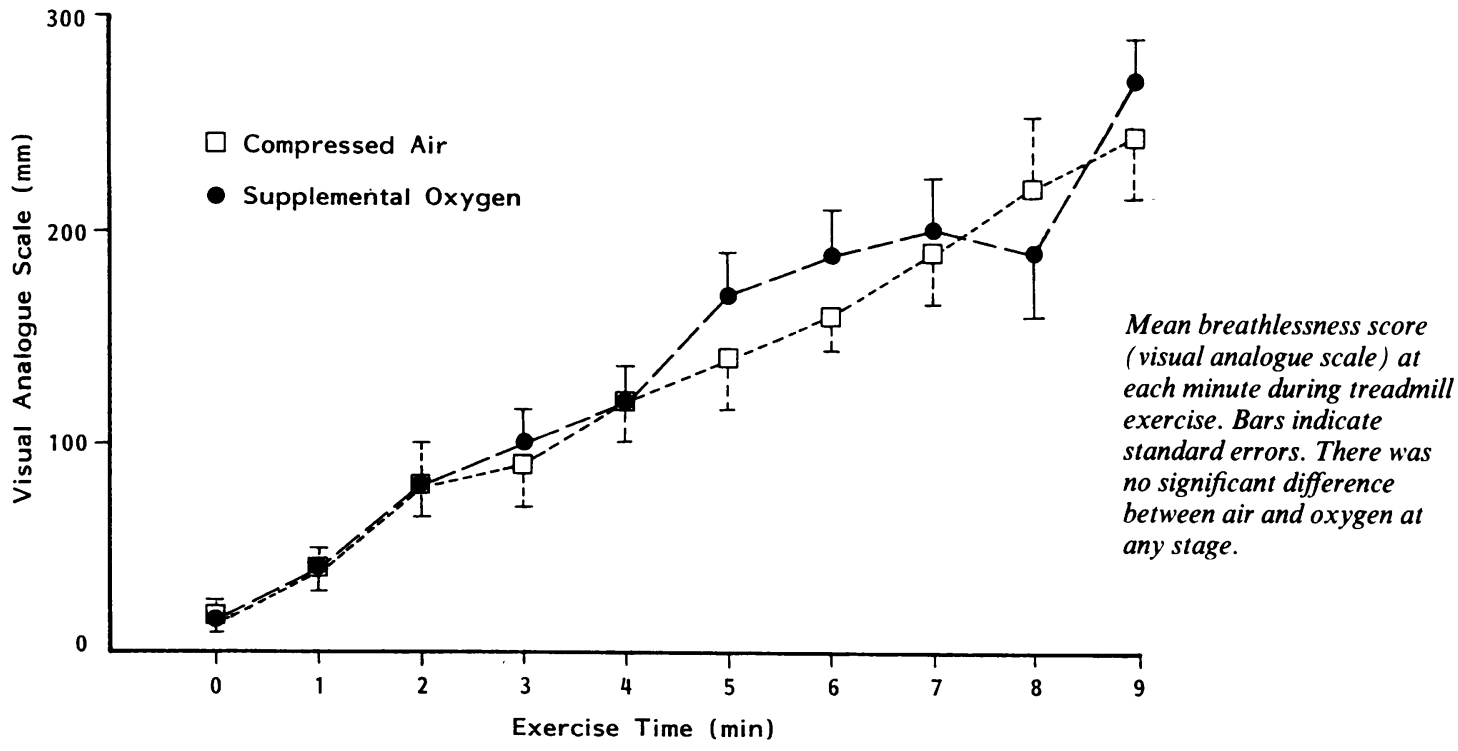

When the order effect of the tests was examined (table 3), there was no significant difference between the first and the second tests for $\mathrm{SaO}_{2}$ at rest or end exercise, but patients walked further in the second test (288 compared with $259 \mathrm{~m}$ ), and HR75 and VAS75 were lower in the second test; the differences were not, however, significant at the 0.05 level.

\section{Discussion}

In 20 patients with chronic obstructive lung disease we were unable to detect a significant improvement in maximal exercise performance or breathlessness during exercise after supplemental oxygen before exercise. This is a contrast to Woodcock et al,' who studied the effect of supplemental oxygen from nasal prongs at $41 \mathrm{~min}^{-1}$ for five minutes before treadmill exercise in 10 patients with chronic obstructive lung disease (mean $\mathrm{FEV}_{1} 0.71$, resting $\mathrm{PaO}_{2} 73 \mathrm{~mm} \mathrm{Hg}(9.7$ $\mathrm{kPa})$ and $\left.\mathrm{PaCO}_{2} 34 \mathrm{~mm} \mathrm{Hg}(4.5 \mathrm{kPa})\right)$. They showed

Table 3 Results (means with standard errors in parentheses) of exercise tests in order of performance

\begin{tabular}{lll}
\hline & lst test & 2nd test \\
\hline Resting $\mathrm{SaO}_{2}(\%)$ & $92(1)$ & $92(1)$ \\
End exercise $\mathrm{SaO}_{2}(\%)$ & $84(2)$ & $83(2)$ \\
DISTmax (m) & $259(36)$ & $288(39)^{*}$ \\
VAS75 (mm) & $190(13)$ & $173(14)$ \\
HR75 (beats/min) & $124(4)$ & $122(4)$ \\
HRmax (beats/min) & $127(5)$ & $129(4)$ \\
\hline
\end{tabular}

*Oxygen significantly different from compressed air by paired $t$ test; $p<0.05 ; 95 \%$ confidence limits of difference between means $1-56$ metres.

VAS75-visual analogue scale breathlessness score at $75 \%$ of maximum distance walked. For other abbreviations see table 2 . that pre-exercise supplemental oxygen produced a small increase in distance walked-from $203 \mathrm{~m}$ to $223 \mathrm{~m}$-accompanied by a small improvement in dyspnoea.

The present study differed from that of Woodcock et al in several ways. Firstly, although mean age and $\mathrm{FEV}$, were similar, our group had a lower mean $\mathrm{PaO}_{2}$ and higher $\mathrm{PaCO}_{2}$. This is unlikely, however, to explain the lack of effect of oxygen in our study since oxygen had no effect in the 11 patients whose characteristics were similar to the 10 patients studied by Woodcock and associates $\left(\mathrm{SaO}_{2}>90 \%, \mathrm{PaCO}_{2}<43 \mathrm{~mm} \mathrm{Hg}\right)$. Secondly, our exercise protocol used linear increases in speed on the treadmill, whereas a protocol of logarithmic increases in speed was used in the Woodcock study. This meant that our patients spent about one and a quarter minutes more on the treadmill. It could be argued that our exercise protocol militated against finding an effect of pretreatment with oxygen or maximum distance walked because of the longer exercise time. We found no differences between air and oxygen, however, in terms of mean breathlessness score or heart rate at any stage during the test. Thirdly, the dosing schedule for oxygen differed between the studies. We chose a flow rate of $2.51 \mathrm{~min}^{-1}$ for 10 minutes for the practical reason that low flow gauges permitting a maximum flow rate of $2.51 \mathrm{~min}^{-1}$ are uniformly prescribed in our region for patients using oxygen at home. This difference in dosing schedule is unlikely to have led to differences in oxygen stores at the start of exercise between the two studies. Lastly, we studied only treadmill exercise, whereas Woodcock $e$ al studied both treadmill walking and six minute corridor walking. Although the six minute 
walk more closely mimics the patients' exercise at home, Woodcock et al found that pre-exercise supplemental oxygen increased the distance walked on both tests, but that the breathlessness score improved only in the treadmill test. The differences between the findings of the two studies are therefore unlikely to be explained by methological differences.

Our findings agree with those of Rhind et al, ${ }^{6}$ who found no improvement in six minute walking distance or heart rate in 12 patients with chronic bronchitis $\left(F_{1} V_{1} 0.56\right.$ l) given supplemental oxygen for 30 minutes before exercise. In this study patients were also given supplemental oxygen or compressed air during recovery, but no reduction in recovery time was found with supplemental oxygen.

One of our authors (JLMcK) has previously studied the effects of breathing supplemental oxygen during exercise in 21 patients with chronic obstructive lung disease, using the same treadmill protocol as in the present study. ${ }^{4}$ In that study mean maximum distance walked was significantly greater with supplemental oxygen $(366 \mathrm{~m})$ than with compressed air $(284 \mathrm{~m})$. On the basis of this finding we decided to design a study capable of detecting an increase of 50 metres in maximum distance walked. With an alpha error of $5 \%$ and a sample size of 20 patients, the beta error was $7.4 \%$ for detection of such a difference between compressed air and supplemental oxygen. Thus the power of the study to detect a clinically significant improvement in maximum distance walked was greater than $90 \%$.

We found an order effect for the studies, with better performance in the second study. Examination of the $95 \%$ confidence limits, however, for the difference between compressed air and oxygen in maximum distance walked shows that the magnitude of this difference was small (table 3). Eleven patients had supplemental oxygen before the second study, which might have biased the study towards showing "improvement" after oxygen. Despite this, no such improvement was found.

We conclude that pretreatment with supplemental oxygen for 10 minutes before progressive maximal exercise does not improve maximum exercise performance or breathlessness during progressive exertion in patients with chronic obstructive lung disease.

We thank Mrs Lyn Jeffrey and Mrs Joy Peate for secretarial assistance, Mr Steven Gyulay, Mr Andrew Coates and Mrs Pam Pratt for technical assistance and Dr Dianne O'Connell for statistical advice.

\section{References}

1 Woodcock AA, Gross ER, Geddes DM. Oxygen relieves breathlessness in "pink puffers." Lancet 1981;i:907-9.

2 Bye PTP, Esau SA, Levy RD, et al. Ventilatory muscle function during exercise in air and oxygen in patients with chronic airflow limitation. Am Rev Respir Dis 1985;132:236-40.

3 Leggett RJE, Flenley DC. Portable oxygen and exercise tolerance in patients with chronic hypoxic cor pulmonale. Br Med J 1977;2:84-6.

4 McKeon JL, Tomlinson JC, Tarrant PE, Mitchell C. The assessment of portable oxygen in patients with chronic obstructive pulmonary disease. Aust NZ J Med 1986; 16:621.

5 Cochrane GM, Prior JG. Management of chronic bronchitis and emphysema. The role of bronchodilators. In: Clark TJH, Cochrane GM, eds. Bronchodilator therapy: the basis of asthma and chronic obstructive airways disease management. Baltimore: Williams and Wilkins, 1984.

6 Rhind CB, Prince KL, Scott W, Flenley DC. Symptomatic oxygen therapy in hypoxic chronic bronchitis. Thorax 1986;41:245.

7 Bond A, Lader M. The use of analogue scales in rating subjective feelings. Br J Med Psychol 1974;47:211-8.

8 Colton R. Statistics in medicine. Boston: Little, Brown and Company, 1974:142-6. 